\title{
O RETORNO DO INVESTIMENTO NA GESTÃO DO CONHECIMENTO COM FOCO NO CAPITAL HUMANO
}

\author{
Marta Silva Neves $\mathbf{1}$; \\ Mônica Pagno da Silva da Rosa; \\ Patrícia de Sá Freire ${ }^{3}$;
}

\begin{abstract}
This study aims to identify the limitations and possibilities of establishing strategies and indicators to measure the Return on Investment (ROI) in relation to Knowledge Management (KM), by means of an integrative review. The articles selected through a search in the Scopus database underwent bibliometric and descriptive analyses, with a focus on measuring ROI, on the metrics they used, and on theories and practices regarding indicators to measure ROI in relation to KM. The study led access to articles that deal with the importance of KM in organizations, but not with its return in terms of monetary value. As a limitation of this study is the little presence of the use of indicators to measure the monetary value of return in relation to the monetary value invested in KM. For future studiesthe theoretical approaches and indicators adopted in the selected articlesare presented, as well asthe research trends in return on investment.
\end{abstract}

Keywords:Knowledge Management; Return on investment; Human capital.

\section{Resumo}

Este estudo propõe identificar as limitações e as possibilidades de estabelecer estratégias e indicadores para aferir o ROI em relação à $\mathrm{GC}$, por meio de revisão integrativa. Através de busca na base Scopus, os artigos selecionados passaram por análises bibliométrica e descritiva, com foco na mensuração do ROI, nas métricas utilizadas e nas teorias e práticas a respeito de indicadores para aferir o ROI em relação à GC. O estudo favoreceu acesso a artigos que tratam da importância da GC nas organizações, mas não o seu retorno em termos de valor monetário. Como limitador deste estudo está a pouca presença do uso de indicadores para mensurar o valor monetário de retorno em relação ao valor monetário investido em GC. Para estudos futuros, apresentam-se as tendências da pesquisa em retorno do investimento, as abordagens teóricas e os indicadores adotados nos artigos selecionados.

Palavras-chave: Gestão do Conhecimento; Retorno sobre investimento; Capital Humano

\footnotetext{
${ }^{1}$ Programa de Pós-graduação em Engenharia e Gestão do Conhecimento - Universidade Federal de Santa Catarina (UFSC) Florianópolis - Brasil - martaneves242@gmail.com

${ }^{2}$ Programa de Pós-graduação em Engenharia e Gestão do Conhecimento - Universidade Federal de Santa Catarina (UFSC) Florianópolis - Brasil - monicapagno@yahoo.com.br

${ }^{3}$ Programa de Pós-graduação em Engenharia e Gestão do Conhecimento - Universidade Federal de Santa Catarina (UFSC) Florianópolis - Brasil - patriciadesafreire@ gmail.com
} 


\section{INTRODUÇÃO}

Cada vez mais, o conhecimento pode ser considerado um recurso fundamental para o desenvolvimento e competitividade da empresa. O processo eficaz do conhecimento, como afirmam Argote e Ingram (2000) e Wiig (1993), possibilita uma base fundamental para a competitividade e a sobrevivência das organizações.

O conhecimento, a partir de sua criação, perpassa pela interação contínua dos níveis individuais, de grupos e da organização, e para tal, Nonaka, Byosicrc, e Borucki (1994) destacam quatro modos de conversão do conhecimento - socialização, externalização, combinação e internalização.

A socialização do conhecimento acontece por meio de interações do indivíduo, incluindo observação, imitação ou aprendizagem, enquanto o conhecimento tácito acontece através do "conhecimento em interações face a face, naturais e tipicamente sociais" (Dalkir, 2005, p. 54). A externalização desse conhecimento do indivíduo por meio de metáforas, analogias ou esboços "fornece uma forma visível para o conhecimento tácito e o converte em conhecimento explícito; o conhecimento anteriormente tácito pode ser anotado, gravado, desenhado ou tornado tangível ou concreto de alguma maneira" (Dalkir, 2005, p. 54-55). A combinação desse novo conhecimento ocorre por meio de diálogos, reuniões, sistemas de informação, tornando-se sistêmico. Nenhum novo conhecimento é criado por si; "é uma nova combinação ou representação de conhecimento existente ou já explícito" (Dalkir, 2005, p. 55). A internalização desse conhecimento sistematizado pelos indivíduos, novo conhecimento, é então utilizado pelos funcionários ou colaboradores que o ampliam, "estendem e reformulam dentro de suas próprias bases de conhecimento tácito, tornando-se uma mudança observável” (Dalkir, 2005, p. 56).

Nessa perspectiva, a Gestão do Conhecimento considera processos que propiciem criação, armazenamento, transferência e aplicação do conhecimento, impulsionando e mantendo a vantagem competitiva da empresa, como destacado por Nonaka e Takeuchi (1995), Nemati, Steiger, Iyer e Herschel (2002). Dessa forma, pode-se afirmar que o processo de criação de conhecimento dentro de uma organização pode se dar por meio de aprendizado, pesquisa e desenvolvimento, acumulação de experiência e aprendendo fazendo. Chang e Lee

\section{(TECNOPUC}


que a capacidade de obter conhecimento pode afetar positiva e significativamente a inovação administrativa e técnica do conhecimento.

Os indicativos da gestão do conhecimento são destacados por Serrat (2017), entre esses: produtos e serviços de conhecimento são estratégicos e devem ser contabilizados e avaliados de acordo; gerenciamento do conhecimento requer integração e equilíbrio de liderança, organização, tecnologia e aprendizado em um ambiente de toda a organização; gestão do conhecimento deve atender aos requisitos e condições de sucesso e aos benefícios e expectativas desejados da organização; a cultura organizacional afeta a gestão do conhecimento, especialmente nos níveis mais baixos; organizações simplificadas com fortes culturas organizacionais têm uma chance maior de sucesso no gerenciamento do conhecimento; atmosfera de confiança é uma pré-condição para o compartilhamento de conhecimento; propostas de gerenciamento de conhecimento devem incluir medidas flexíveis e rígidas para que os gerentes apoiem iniciativas de gerenciamento de conhecimento.

Ao mesmo tempo, há que se considerar que os processos, ferramentas e metodologias de gestão do conhecimento são referentes a cada contexto organizacional, considerando todas as circunstâncias, pois necessitam de recursos de suporte organizacional e um contexto de estímulo (Gold, Malhorta e Segars , 2001).

Nessa perspectiva, o conhecimento representa uma fonte para o aprendizado e desenvolvimento organizacional, sendo que os processos de aquisição, compartilhamento e aplicabilidade do mesmo levam a implementar a gestão enquanto capital intelectual e ativo intangível, por seu valor diferenciado.

A gestão dos ativos intangíveis vem a ser o objetivo da Gestão do Conhecimento, aprimorando a criação, aquisição e integração ao valor da organização.

Os ativos intangíveis, por serem abstratos, tornam-se desafios para a medição e gerenciamento, e estão relacionados ao valor econômico e social de uma empresa (O’Donnell, O’Regan e O’Regan, 2000). Ao mesmo tempo, são ativos que agregam valor ao negócio corporativo por serem "únicos, difíceis de adquirir, de desenvolver e até mesmo de copiar, além disto, alguns podem ser até protegidos legalmente” (Perez e Famá, 2006, p. 84).

Desta forma, tem-se o capital intelectual e corroborando com o exposto, BuenecheaElberdin, Kianto e Sáenz (2017) defendem que os ativos de conhecimento valioso que a 
corporação detem, gerencia ou tem acesso com o objetivo de alcançar vantagens competitivas corresponde ao capital intelectual.

O capital intelectual é composto por três dimensões identificadas por Dzinkowski (2000) - capital humano; capital estrutural; capital relacional.

O capital humano é relativo às competências das pessoas, suas capacidades, habilidades e especializações dos recursos humanos que integram à organização (Lynn, 2000). Para que esse capital humano beneficie toda a empresa é necessário que seja compartilhado e transmitido, contando com estruturas internas como laboratórios, sistemas de informações, canais de distribuição, que caracterizam o capital estrutural. A partir das relações estabelecidas entre a entidade e as pessoas que mantêm operações, caracteriza-se o capital relacional ou de clientes (Stewart,1998).

Nesse sentido, gerenciar o capital intelectual implica em aproveitar o capital humano, isto é, gerenciar as competências individuais para criar valor (Edvinsson e Sullivan, 1996).

O capital humano, conforme Schmidt e Santos (2002), são os benefícios proporcionados pelos indivíduos à instituição, que exige esforço na identificação de pessoas para interagir na organização e otimizar esse "ativo humano", que tem seu valor acumulado de investimento em treinamento, competências, relacionamentos e valores pessoais.

O capital humano está relacionado mutuamente com os capitais estrutural e relacional, pois, à medida que as pessoas e a empresa aprendem e se desenvolvem, impulsionam-se o propósito, a agilidade, o gerenciamento, a interação e o relacionamento com clientes, fornecedores e a outros com quem se relaciona.

Nesse sentido, a revisão de literatura apresentada neste artigo examina a correlação da gestão do conhecimento, capital humano e retorno do investimento, estabelecendo um recorte a respeito de indicadores e modelos que interpretem esse ativo intangível com o objetivo de identificar as limitações e as possibilidades de estabelecer estratégias e indicadores para aferir o ROI em relação à GC.

\section{PROCEDIMENTOS METODOLÓGICOS}


Para atingir o objetivo do estudo - identificar as limitações e as possibilidades de estabelecer estratégias e indicadores para aferir o ROI em relação à GC - esta pesquisa foi realizada de forma exploratória descritiva, por meio de revisão integrativa.

A abordagem metodológica se caracteriza como quantitativa com análise bibliométrica; e, também, qualitativa, com análise descritiva sobre os objetivos e propósitos das publicações levantadas.

Assim, a revisão baseou-se na análise do conhecimento científico existente sobre "Gestão do Conhecimento", "Retorno sobre o investimento" e "Capital Humano". Para tal, o estudo perpassou pela definição dos temas, elaboração da pergunta de pesquisa - quais as métricas utilizadas para a mensuração do retorno sobre o investimento em gestão do conhecimento? - busca na base de dados; categorização dos estudos, mediante filtragem dos dados; análise dos estudos incluídos; discussão dos resultados, finalizando com a apresentação da revisão integrativa.

Ao definir os objetivos e pergunta de pesquisa, foram identificados os termos em inglês: "knowledge management" e "return on investiment" ou "investment in knowledge management" ou "knowledge management value" ou "human capital" para a pesquisa na base de dados, definindo estratégias quanto ao campo de busca, filtragem e resultados prévios.

O campo de busca escolhido foi a base Scopus, por ser a maior base de dados com resumo e citações de artigos científicos (Falagas, Pitsouni, Malietzis e Pappas, 2008 ). Para tal, foram utilizados os conceitos-chave - gestão do conhecimento, retorno sobre o investimento e capital humano, tendo como estratégias de busca title - abs - key, que resultou em 264 artigos. Para a filtragem considerou-se o período de publicações a partir do ano de 2010 e documentos do tipo artigo.

Os resultados prévios da pesquisa apontaram 264 artigos publicados no recorte temporal entre 2010 a 21 de maio de 2020, assim distribuídos, 124 publicações entre 2010 e 2015 e 140 publicações entre 2016 até a data limite de 2020, demonstrando o interesse e atualidade do tema nos últimos cinco anos. Desses 264 artigos, a partir da leitura dos resumos e palavras-chave, foram selecionados 92 artigos. Dos 92 selecionados, se obteve acesso a 36 artigos para leitura na íntegra. 


\section{APRESENTAÇÃO E ANÁLISE DE RESULTADOS}

Após a seleção dos 92 artigos, passou-se às análises bibliométrica e descritiva. A análise bibliométrica foi realizada com uso da base de dados Scopus e do software Microsoft Excel. Para a análise descritiva foram consideradas as leituras dos 36 artigos. Os artigos foram analisados de acordo com os objetivos estabelecidos previamente, considerando: como as empresas medem o ROI sobre a GC, as métricas utilizadas pelas empresas para aferir o ROI em relação à GC e as limitações e possibilidades de estabelecer indicadores para aferir o ROI em relação à GC. Após a análise dos dados e comparação dos artigos, foram apresentadas as considerações na seção conclusão.

A seguir são apresentados os resultados das análises bibliométrica e descritiva.

\subsection{ANÁLISE BIBLIOMÉTICA}

O levantamento obtido com os procedimentos metodológicos resultou na seleção de 92 documentos, caracterizados como artigos. Os primeiros critérios utilizados para a seleção dos documentos foi a leitura do resumo e palavras-chave de cada um. Dos 92 artigos analisados, 77 apresentaram acesso fechado e 15 livre acesso. Mesmo assim, se obteve acesso para leitura a 36 artigos na íntegra, por meio de disponibilização e solicitação direta aos autores via plataforma ResearchGate.

A multidisciplinariedade dos estudos está retratada em diversas áreas de conhecimento relacionadas nos artigos levantados, destacando-se entre elas de forma decrescente: Negócios, Gestão e Contabilidade (34,3\%); Engenharia (12,7\%); Ciência da Computação (12\%); Ciências Sociais (11,4\%). Destaca-se a interdisciplinaridade nos 92 artigos, correlacionando duas ou mais áreas resultantes, pois a soma de todas as áreas chega a 166. Vale destacar uma das áreas com menor incidência de artigos: Economia, Econometria e Finanças, apenas 5,4\%. Isso demonstra a carência de pesquisas que envolvem essa área.

A quantidade de publicações por ano, retratada no Gráfico 1, demonstra a constância de interesse pelo tema, com pico de publicações no ano de 2014, abrangendo diferentes áreas do conhecimento e fontes de publicação. É possível observar que após o ano de 2014 houve 
um decréscimo no número de publicações, atingindo seu menor valor no ano de 2017. Ainda o estudo do tema pesquisado ganhou relevância nos anos de 2011 e 2014, pois são os anos que apresentam um número de publicações expressivo.

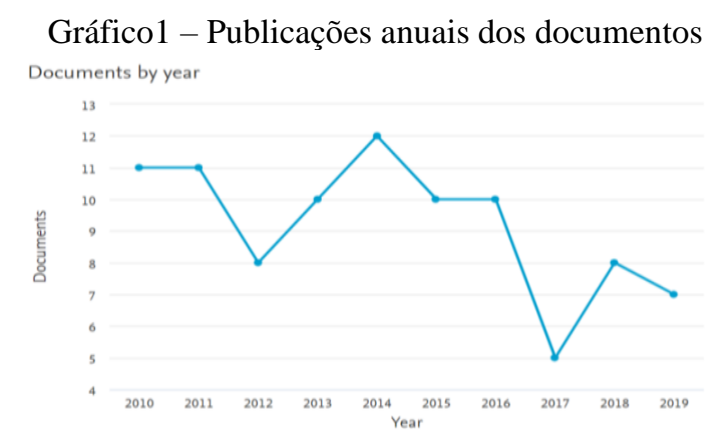

Fonte: Scopus (2020)

O levantamento demonstra as cinco fontes com maior número de publicações, sendo importante destacar a presença do "Journal of intelectual Capital". Essa fonte publica pesquisas sobre criação, identificação, gerenciamento, medição e proteção de fatores que determinam o valor de uma organização, incluindo capital relacional, estrutural e humano. Destaca-se o capital humano, tema presente nesta pesquisa.

O Gráfico 2, ressalta os Estados Unidos como país com o maior número de publicações, 11 publicações, seguido por Austrália com 10 publicações.

O Brasil não se destaca na lista de fontes das publicações, Gráfico 2, e não apresenta um número de publicações relevante (3 publicações) para destaque no Gráfico 2. Há uma diversidade em relação aos meios de publicação dos artigos selecionados, todavia, a maioria foi publicada em revistas, anais e livros.

Gráfico 2 - Número de publicações por país 


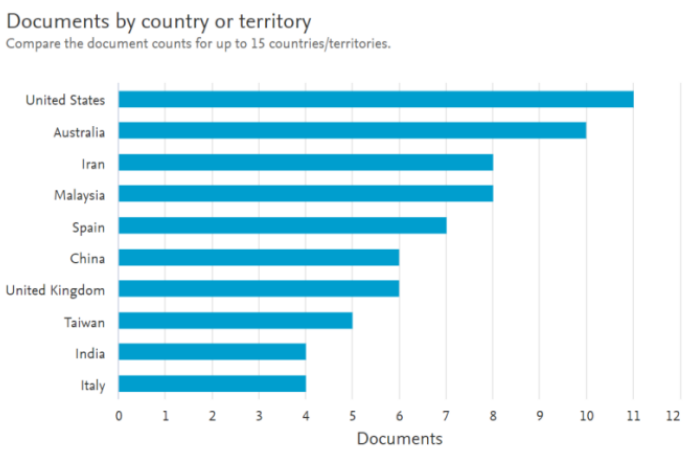

Fonte: Scopus (2020)

Por fim, o mapeamento bibliométrico permitiu identificar a relação de artigos mais citados. O número mais alto é 154 citações para o mesmo artigo, cujo autor é Guerrero, M., da Universidade de Deusto, Espanha. Seguido de 105 citações alcançadas pelo segundo artigo mais citado, de autoria de Martínde Castro, G., da Universidade Complutense de Madri, Espanha.

\subsection{ANÁLISE DESCRITIVA}

Para a leitura dos 36 artigos, acessados na íntegra, foram adotadas três perguntas norteadoras: frente a dificuldade de medir o ROI, como esse é mensurado?; quais as métricas utilizadas para aferir o ROI e GC?; quais as teorias e práticas que demonstram limitações e possibilidades pra estabelecer indicadores para aferir o ROI em relação à GC?

O cenário e o público-alvo dos artigos estão representados por instituições financeiras, organizações não governamentais (ONGs), empresas privadas e públicas, empresas de tecnologia, logística, biotecnologia, telecomunicações, hotelaria, terceirização de serviços, pequenas e médias empresas, universidades, indústrias e indústria farmacêutica.

O foco de atenção da primeira pergunta está voltado à dificuldade de medir o ROI e como esse é mensurado, o que favoreceu identificar os seguintes aspectos: a adoção de técnicas para GC para acúmulo do capital intelectual para lidar com constantes mudanças (Shih, Chang e Lin,2010); a implementação de ferramentas de TI, podendo ou não alcançar os resultados almejados (Anantatmula e Kanungo, 2006); a captura e a retenção do conhecimento organizacional, reduzindo a potencial perda de capital humano; o 
acompanhamento das práticas de GC como manutenção de repositórios, desenvolvimento de comunidades de prática, inteligência de negócios e aprendizado contínuo no desempenho organizacional (Abdullah, Isa, Arshad, Hamzah e Hashim, 2013; Gantasala, Padmakumar, Yasin e Gantasala, 2010); o foco na formação pessoal para atingir metas corporativas, a partir da criação de ambiente favoráveis ao desenvolvimento do potencial humano e partilha de conhecimentos e habilidades (Henard e McFadyen, 2012; Suraj e Ajiferuke, 2013); a atenção à influência do indivíduo nos processos de GC (Gubbins et al., 2012); o domínio da tecnologia para mais informações, flexibilizando tomada de decisões e a maior competitividade da organização (Chang, 2013).

Ainda, em resposta à primeira pergunta, de acordo com os artigos lidos, o ROI pode ser mensurado através da conexão entre elementos organizacionais e a eficácia da GC, investindo no desenvolvimento de sistemas de GC (Biloslavo, Kljajic-Dervic e Dervic, 2018); do investimento em tecnologia da informação, investimento em treinamento de pessoal, investimento em gestão do conhecimento e aprimoramento das práticas administrativas das empresas (Arévalo-Avecillas, Nájera-Acuña e Piñero, 2018); do desenvolvimento de mais componentes nos sistemas de gerenciamento de conhecimento (Mishchuk, Bilan, e Pavlushenko, 2016).

As métricas utilizadas para aferir o ROI e GC nos documentos estudados foi a segunda questão a ser investigada, que propiciou um recorte bem diverso de estratégias, principalmente focadas em gerenciamento do conhecimento para acúmulo do capital intelectual, por meio de metas específicas, mensuráveis e realizáveis para motivar funcionários ao desempenho superior (Gantasala et al.,2010). Identifica-se a atenção a um processo sistemático de busca, seleção, organização, concentração e apresentação de conhecimentos para aumento do nível de entendimento dos funcionários relacionado a áreas específicas das organizações (Antosová, 2010). Nesse sentido, identificaram-se métricas objetivas como, porcentagem de funcionários envolvidos em compartilhamento de conhecimento (Teo, Nishant, Goh e Agarwal, 2011), avaliação de desempenho dos funcionários e o desenvolvimento de competências, número de documentos criados em repositórios de informações, acesso a blogs, aumento em tópicos de discussão, postagens em blogs, sendo a atenção voltada ao maior nível de interação. Destacam-se também, o uso de 
taxas de investimento, taxa de crescimento do investimento, taxa de vendas, taxa de crescimento de vendas, bem como, a participação no mercado e a taxa de crescimento, como índices de desempenho (Lee, 2011).

Vale ressaltar que a eficácia na GC influencia o desempenho dos negócios da empresa, tanto nos indicadores financeiros, como não financeiros (Biloslavo et al., 2018). O investimento em tecnologia da informação, investimento em treinamento de pessoal, investimento em gestão do conhecimento e aprimoramento das práticas administrativas das empresas, podem apresentar uma influência significativa e positiva na lucratividade das empresas (Arevalo-Avecillas et al., 2018). As empresas que desenvolveram mais componentes nos sistemas de gerenciamento de conhecimento apresentam maior lucratividade e proporção entre salário médio mensal na empresa e no setor (Mishchuk et al., 2016).

A leitura dos documentos pesquisados favoreceu identificar teorias e práticas que demonstram limitações e possibilidades de estabelecer indicadores para aferir o ROI em relação à GC, conforme destacou Cohen (2006).

Percebem-se estudos que demonstram a dificuldade em avaliar o ROI em determinados sistemas de GC, em virtude do estágio evolutivo do mesmo (Anantatmula e Kanungo, 2006). Ao mesmo tempo, reconhece-se que a implementação bem sucedida de GC favorece o êxito nos indicadores de tarefa, processo e organização, que contribuem na mensuração da melhoria de processos, levando à redução de custos e vantagem competitiva.

Outro aspecto percebido nos artigos estudados é a Governança da GC (Zyngier,2011; Zyngier e Burstein, 2012), enquanto meio eficaz para alcance de benefícios e fomento aos recursos de conhecimento organizacional, por meio de estratégias regulamentadas de GC. Embora, algumas pesquisas apontem falta de clareza ao medir e avaliar as estratégias de GC, por conta de diversos objetivos e metas, identificam-se a adoção de critérios que podem estabelecer o ROI nas estratégias de GC, entre eles: análise do crescimento do capital humano (Liebowitz e Wright, 1999); o uso do Balanced Scorecard (Kaplan e Norton, 2001), incluindo medidas financeiras, de crescimento e de clientes, e processos internos de negócios; a avaliação dos objetivos, normativas, operacionais e estratégicas das estratégias de GC (Probst, Raub e Romhardt, 2000). 
Entre outros modelos de avaliação das estratégias de GC está o de Kirkpatrick (1994) e Phillips (1997), compreendendo cinco níveis. O primeiro nível corresponde a mensuração de reações dos alunos por meio de dados operacionais diretos. O nível seguinte está relacionado ao comportamento de aprendizagem. O terceiro nível trata da transferência de aprendizagem para o local de trabalho. O nível quatro aborda o impacto nas métricas de negócio e o nível cinco trata de dados de retorno de investimento (Gubbins et al., 2012).

A implementação de métricas multiníveis também está presente no gerenciamento de iniciativas para partilha de conhecimento nas organizações (Teo et al., 2011; Suraj e Ajiferuke, 2013; Masa'deh, Maqableh e Karajeh, 2014). Os estudos buscam foco consistente e com responsabilidade adequada, caso iniciativas falhem, abrangendo avaliações de lideranças como ponto impulsionador e avaliações de equipes. $\mathrm{O}$ destaque da liderança vai para o desenvolvimento de lideranças por meio de intervenções como orientação, treinamento, aprendizado ativo, programas intensivos de feedback, entre outros (Day, 2001).

Entre as pesquisas, estão os esforços das organizações em altos investimentos na educação de habilidades dos funcionários, na estrutura da empresa e na conexão com os clientes, a fim de promover pesquisa e desenvolvimento (P\&D), refletindo no desempenho financeiro futuro (Lee, 2011; Chang, 2013; Henard e McFadyen, 2012; Abdullah et al., 2013; Almasarweh, Alnawaiseh, Alsaraireh e Al Wadi, 2019). Para tal, os gerentes implementam investimentos nessas dimensões do capital intelectual, que a partir da análise de variáveis desenvolvem o demonstrativo de eficiência do capital humano, do capital estrutural, do capital relacional e esses, impactando na tecnologia da empresa. A eficiência do capital tecnológico torna-se um mediador entre o capital intelectual e o desempenho financeiro da empresa.

No contexto de outros estudos, apresentam-se os procedimentos de documentação da ISO 9000, a fim de codificar o conhecimento tácito em conhecimento explícito. As experiências identificaram como benefícios: oportunizar consistências nos processos, controle, orientações para treinamento e favorecer a melhoria contínua (Antosová, 2010; Ataseven, Prajogo e Nair, 2014). As pesquisas apontaram a necessidade de equilíbrio entre o conhecimento e o trabalho propriamente dito, considerando que o armazenamento do conhecimento, rotinas e estruturas de rede são ativos potenciais para as organizações.

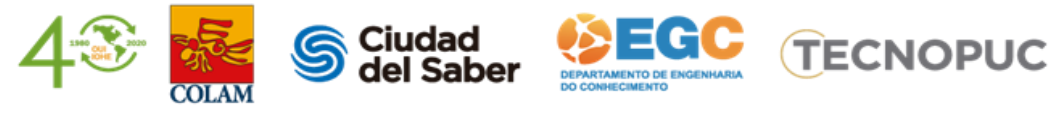




\section{CONCLUSÃO}

As métricas identificadas na leitura dos 36 artigos são essencialmente não-financeiras e alicerçadas nos conceitos de organizações do conhecimento, não apresentando mensuração, explícita, quanto ao retorno do investimento sobre a GC. Ao mesmo tempo, os artigos demonstram a importância das organizações adotarem uma GC eficaz e eficiente, bem como, seus benefícios como um todo.

O capital humano, suas competências, habilidades, conhecimentos, comprometimento, o compartilhamento do conhecimento contribuem para o sucesso da implementação da GC. Mas, para isso, é imprescindível que as organizações invistam em treinamentos e desenvolvimento pessoal de seus colaboradores.

O investimento e adoção de tecnologias da informação pelas organizações, no processo de GC, apresentam relevância, mas são um meio, uma forma de auxílio à GC e não a solução efetiva dos problemas, por si só. Pois, neste contexto, sem uma GC eficaz e eficiente as tecnologias da informação, sozinhas, nada podem fazer.

Os estudos levantados e a revisão teórica demonstram que a governança de GC vem ao encontro da mensuração da GC e do ROI, representando um meio regulamentado para certificar o alcance dos benefícios propostos com a GC. Mas, para que isso seja possível, se faz necessária a adoção de métricas claras e objetivas que possam mensurar, de fato, qual é o ROI sobre a GC.

Embora existam indicadores e instrumentos para avaliar o capital intelectual da GC, identificou-se como limitador deste estudo a pouca presença da relação ROI e GC nos artigos analisados, ou seja, indicadores claros que mensurem o valor monetário de retorno em relação ao valor monetário investido em GC. O estudo proporcionou acesso a uma quantidade expressiva de artigos tratando a importância da GC nas organizações, mas não o seu retorno em termos de valor monetário.

Para trabalhos futuros, sugere-se a criação de métricas e indicadores para mensuração do ROI sobre a GC quanto ao valor monetário e aplicação dos mesmos em uma organização, para que possam ser validados. 


\section{REFERÊNCIAS}

Abdullah, N. L., Isa, R. M., Arshad, R., Hamzah, N., \& Hashim, N. A. (2013) Knowledge management strategy and human capital management in hotel industry. Jornal Pengurusan, 38, 3-14.

Almasarweh, M.S., Alnawaiseh, M.B., Alsaraireh, A.A. \& Al Wadi, S. (2019) Intellectual capital and effect on marketing performance an empirical study in Jordania pharmaceutical industrial firms. Italian Journal of Pure and Applied Mathematics, 42, 954-964.

Anantatmula, V. \& Kanungo, S. (2006) Estruturando as relações subjacentes entre os resultados da gestão do conhecimento. Journal of Knowledge Management, 10 (4), $25-42$.

Antosová, M. J. (2010) Human resources management and organizational development as a basis for the knowledge management. Acta Montanistica Slovaca, 15 (1), 90-95.

Arévalo-Avecillas, D., Nájera-Acuña, S. \& Piñero, E. A. (2018) La influencia de la implementácion de las tecnologías de información em la productividad de empresas de servicios. Informácion Tecnológica, 29 (6).

Argote, L., \& Ingram, P. (2000) Knowledge transfer: a basis for competitive advantage in firms. Organizational Behavior and Human Decision Processes, 82 (1), 150-169.

Ataseven, C., Prajogo, D. I. \& Nair, A. (2014) ISO 9000 internalization and organizational commitment - implications for process improvement and operational performance. IEEE Transactionson Engineering Management, 61 (1), art. n. 6655906, 5-17.

Buenechea-Elberdin, M., Kianto, A. \& Sáenz, J. (2017) Intellectual capital drivers of product and managerial innovation in high-tech and low-tech firms. R\&D Management, 48 (3). https://doi.org/10.1111/radm.12271

Biloslavo, R., Kljajic-Dervic, M. \& Dervic, S. (2018) Factors affecting e effect iveness of knowledge management: a case of Bosnia and Herzegovina trade enterprises. Knowledge Process Management, 1-12. https://doi.org/10.1002/kpm.1570

Chang, W. S. (2013) Are R\&D and intellectual property rights related to the firms' financial performance? The perspectives on intellectual capital. Internacional Journal of Technology policy and Management.

Chang, S. C., \& Lee, M. S. (2008) A ligação entre capacidade de acumulação de conhecimento e inovação organizacional. Journal of Knowledge Management, 12 (1), 3-20.

Cohen, D. (2006) Qual é o seu retorno sobre o conhecimento? Harvard Business Review.

Dalkir, K. (2005) Knowledge management in theory and practice. Burlington, MA, Elsevier, 54-56. 
Day, D. (2001) Desenvolvimento de liderança: uma revisão no contexto. Leadership Quarterly, 11, 581-613. http://dx.doi.org/10.1016/S1048-9843(00)00061-8

Dzinkowski, R. (2000) The measurement and management of intellectual capital: an introduction. Management Accounting: Magazine for Chartered Management Accountants, 78 (2), 32-36.

Edvinsson, L. \& Sullivan, P. (1996) Desenvolvendo um modelo para gerenciar o capital intelectual. European Management Journal 14 (4): 356-65.

Falagas, M. E., Pitsouni, E. I., Malietzis, G. A., \& Pappas, G. ( 2008 ) Comparação de Pubmed, Scopus, Web of Science e Google Scholar: Pontos fortes e fracos . FASEB Journal, 22 ( 2 ), 338 - 342 .

Gantasala, P. V., Padmakumar, R., Yasin, A., \& Gantasala, S. B. (2010) The role of HR practices and knowledge management onorganizational performance. International Journal of Knowledge, Culture and Change Management, 10 (1), 151-172.

Gold, A. H., Malhorta, A., \& Segars, A. H. (2001) Knowledge management: an organizational capabilities perspective., Journal of Management Information Systems, 18 (1), 185214.

Gubbins, C., Corrigan, S., Garavan, T.N., O'Connor, C., Leahy, D., Long, D. \& Murphy, E. (2012) Evaluating a tacit knowledge sharing initiative: A case study. European Journal of Training and Development, 36 (8), 827-847.

Henard, D.H. \& McFadyen, M.A. (2012) Resource dedication and new product performance: A resource-basedview. Journal of Product Innovation Management, 29 (2), 193-204.

Kaplan, R. S. \& Norton, D. P. (2001) Transformando o Balanced Scorecard de Medição de Desempenho em Gerenciamento Estratégico: Parte 1, Horizontes Contábeis 15 (1), 87-104

Kirkpatrick, D. L. (1994) Avaliando programas de treinamento. Os quatro níveis, BerrettKoehler, São Francisco, CA.

Lee, M.C. (2011) Knowledge-based new product development through knowledge transfer and knowledge innovation. Inovation through Knowledge Transfer, 303-320. https://doi.org/10.1007/978-3-642-14594-0_31

Liebowitz, J. \& Wright, K. (1999) Um olhar para a avaliação do capital humano. Em Liebowitz, J. (Ed.), Knowledge Mangement Handbook 5.1-5.13. Boca Raton, FL: CRC Press.

Lynn, B. E. (2000) Intellectual capital: unearthing hidden value by managing intellectual assets. Ivey Business Journal. Toronto.

Masa'deh, R.M.T., Maqableh, M.M. \& Karajeh, H. A (2014) Theoretical perspective on the relationship between leadership development, knowledge management capability, and firm performance. Asian Social Science, 10 (6), 128-137. 
Mishchuk, H., Bilan, Y. \& Pavlushenko, L. (2016) Knowlwdge management systems: issues in enterprise human capital management implementation in transition economy. Polish Journal of Management Studies, 14 (1).

Nemati, H. R., Steiger, D. M., Iyer, L. S. \& Herschel, R. T. (2002) Knowledge warehouse: an architectural integration of knowledge management, decisions support, artificial intelligence and data warehousing. Decision Support Systems, 33, 143-61.

Nonaka, I., Byosicrc, P. \& Borucki, C. C. (1994) Teoria da criação de conhecimento organizacional: Um primeiro teste abrangente. International Business Review, 3, 337351. https://doi.org/10.1016/0969-5931(94)90027-2

Nonaka, I., \& Takeuchi, H. (1995) The Knowledge-Creating Company: How Japanese Companies Create the Dynamics of Innovation. Oxford University Press, New York, NY.

O'Donnell, D, O'Regan, P., \& O'Regan V. (2000) Recognition and measurement of intellectual capital: the accounting-relating challenges of intellectual capital. Proceedings of the $3^{\text {rd }}$ International Conference on Practical Aspects of Knowledge Management. Based, Switzerland, 30-31.

Perez, M. M.; \& Famá, R. (2006) Características estratégicas dos AIs e o desempenho econômico da empresa. Revista Eletrônica de Gestão de Negócios - eGesta, 2 (2), 6996.

Phillips, J. J. (1997) Retorno do Investimento, GulfPublishing Co., Houston, TX.

Probst, G., Raub, S. \& Romhardt, K. (2000) Gerenciando Conhecimento;Blocos deconstrução para o sucesso, Chichester: John Wiley\& Sons.

Schmidt, P. \& Santos, J. L. (2002) Avaliação de ativos intangíveis. São Paulo: Atlas.

Serrat, O. (2017) Notions of Knowledge Management. Asian Development Bank. Knowledge solutions: tools, methods, and approaches to drive development for ward and enhance its effects. Mandaluyong City, Philippines: Asian Development Bank, Springer Open, 291-304.

Shih, K.H., Chang, C.J., \& Lin, B. (2010) Assessing knowledge creation and intellectual capital in banking industry. Journal of Intellectual Capital, 11 (1), 74-89.

Stewart, T. A. (1998) Capital intellectual - A nova vantagem competitiva das empresas. Rio de Janeiro. Campus.

Suraj, O.A., Ajiferuke, I. (2013) Knowledge management practices in the Nigerian telecommunications industry. Knowledge and Process Management, 20 (1), 30-39.

Teo, T.S.H., Nishant, R., Goh, M.\& Agarwal, S. (2011) Leveraging collaborative technologies to build a knowledge sharing culture at HP analytics. MIS Quarterly Executive, 10 (1), 1-18.

Wiig, K. M. (1993) Knowledge management foundations, Arlington, TX, EUA: Schema Press. 
Ciudad del Saber, Panamá

X Congreso internacional de conocimiento e innovación

Zyngier, S. (2011) Knowledge management: Realizing value through governance. International Journal of Knowledge Management, 7 (1), 35-54.

Zyngier, S., Burstein, F. (2012) Knowledge management governance: The road to continuous benef its realization. Journal of Information Technology, 27 (2),140-155. 